Supplemental Table S1. Primers used in this study.

\begin{tabular}{|c|c|c|}
\hline Primer & 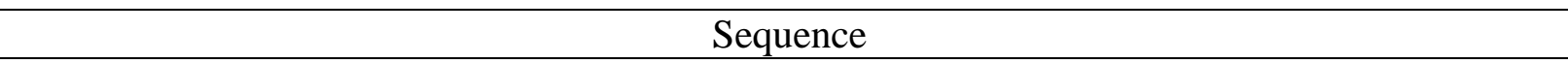 & Primer designation \\
\hline lptenExFw & 5'- ATCAAGTACAAATAATACTTTTGAAATC-3' & $\mathrm{P} 1$ \\
\hline lptenExRV & 5'- АTTTCATTATTTTCAAATACACAATCAC-3' & $\mathrm{P} 2$ \\
\hline lptengenomFW & 5'- AGAAACGACCAAAATTTTACATC-3' & P3 \\
\hline lptengenomRv & 5'- ATTTTAATGAAATAAAATTATCAATA-3' & P4 \\
\hline lptengenomicFw1 & 5'-ATTTTTGTTTCATCTTAATCTGCCATTATTTGTTTGTCACAACATATCATTTTAGCAAA CAATAG-3' & P5 \\
\hline lptengenomicRv1 & 5'- TCTTTTGATGTACCATAAAACGAATTTCCCATACTACC-3' & P6 \\
\hline EZTN-R & 5'- GCCAATATGCGAGAACACCCGAG-3' & P7 \\
\hline lptenFW & 5'- ATGGACTTAGTAAGAAAAATGG-3' & P8 \\
\hline mRFPrv & 5'- TGCACCTGTTGAATGTCTACCTTCTG-3' & P9 \\
\hline IG7-FP & 5'- TTACATTTATTAGACCCGAAACCAAGCG-3' & $\begin{array}{l}\text { Figure 1B, } \\
\text { Amplification of } r n l A\end{array}$ \\
\hline IG7-RP & 5'- TTCССTTTAGACCTATGGACCTTAGCG-3' & $\begin{array}{l}\text { Figure 1B, } \\
\text { Amplification of } r n l A\end{array}$ \\
\hline ptenAcDNAFw & 5'- ATGAGTAATTTATTAAGAGTTGC-3' & $\begin{array}{l}\text { Figure } 1 \mathrm{E} \\
\text { Amplification of ptenA }\end{array}$ \\
\hline ptencDNArv & 5'- ACTTGAGCTATTTGAAGAAG-3' & $\begin{array}{l}\text { Figure } 1 \mathrm{E} \\
\text { Amplification of ptenA }\end{array}$ \\
\hline M13Rv & 5'- CAGGAAACAGCTATGA-3' & $\begin{array}{l}\text { Methods, } \\
\text { Colony PCR to obtain }\end{array}$ \\
\hline
\end{tabular}


lptenRV

5'- TAAGTTGAAGTATTTGTTGTAGTTGT-3'

lptenFWEntry 5 '- caccATGGACTTAGTAAGAAAAATGG-3'

lptencDNArv

lptenFwseq1

lptenFwseq2

lptenFwseq3

lptenFwseq4

lptenFwseq5

lptenFwseq6

lptenFwseq7

\section{5'- CACTTTTTATTTTTAATATTGATAATTTTATTTCAT-3'}

5'- ATGGGTTTTCCTAGTGAATCATTGG-3'

5'- ATGTGGTTGGATCAAAATCC-3'

5'- TGGTTGTGAACCATATTTATCAATTGTTCAACAAGG-3'

5'- AGCACATAAAGATTTAAAACATTTCC-3'

5'- ATGTAATCTTCCTATAACTGCAACTGATCC-3'

5'- ATGAAACCCCAATGGATCC-3'

5'- TGAAAAATCATCATCATCATTATCC-3' lpten-bsr-plasmid

Methods,

Colony PCR to obtain lpten-bsr-plasmid

Methods, Colony PCR D.d.

Methods, Colony PCR D.d.

Methods, Subcloning

of aggregation

comptent lpten cDNA

Methods, Subcloning

of aggregation

comptent lpten cDNA

Sequencing lpten

Sequencing lpten

Sequencing lpten

Sequencing lpten

Sequencing lpten

Sequencing lpten

Sequencing lpten 